mortality. It would simply mean that the cause of death would be different.

Enabling people to live longer-perhaps for 20 years after retirement-would create economic problems no less great than at present. There would perhaps be the compensation that more and more of the unemployed could be used in looking after the aged. Or will you be proposing what the Archbishop of Canterbury hinted at when financial resources really dry up ?

S EllisoN

London NW6

\section{A cautionary tale}

SIR,- 'Tis with a sense of grievous shock That one observed great Stephen Lock To perpetrate the awful sin Of leaving an unchecked reference in An article that bears his name. ${ }^{1}$ This blot on editorial fame Though "For Debate" is clear to see At least to Belloc and to me: It was not Lundy's noble dad Who did advise the tearful lad It was his grandpapa the Duke - Sir, what a liberty you took!

Edinburgh

JACK CORMACK

${ }^{1}$ Lock, S, British Medical fournal, 1976, 2, 1548.

* *Dr Cormack is right, but may I use some more lines of Belloc for my apology?

"When I am dead, I hope it may be said:

'His sins were scarlet, but his books were read.', -ED, $B M 7$.

\section{Management of Hodgkin's disease}

SIR,-Members of the British National Lymphoma Group will have been interested in the paper from St Bartholomew's Hospital on intensive investigation in the management of Hodgkin's disease (4 December, $p$ 1343). The results and conclusions in their paper are remarkably similar to those found in a much larger series of patients reported by the National Lymphoma Group last year. ${ }^{1}$

It is curious that the Barts authors made no mention of this paper in their own report.

SYLVIA WaTkINS

Lister Hospital,

${ }^{1}$ Report from British National Lymphoma Investigation, Clinical Radiology, 1975, 26, 151.

\section{Diffuse myelitis associated with rubella vaccination}

SIR,-The report by Dr S Holt and others ( 30 October, p 1037) is most interesting. My own observations on a similar case may be pertinent.

In April 1975 a 27-year-old married woman came under my care complaining of paraesthesiae of both legs with a mild paraparesis. She had increased reflexes in both legs with a left extensor plantar response and loss of pain and temperature sensation to $T 5$ bilaterally. There was decreased appreciation of vibration and position sense at both ankles. Eight days previously she had been vaccinated with live rubella virus. Her previous medical history and family history were non- contributory. She had an erythrocyte sedimentation rate (ESR) of $48 \mathrm{~mm}$ in the first hour and cerebrospinal fluid (CSF) examination showed clear fluid with $13 \times 10^{6}$ lymphocytes $/ 1\left(13 / \mathrm{mm}^{3}\right)$ and $0.42 \mathrm{~g}$ protein $/ 1$ with $10.4 \%$ gammaglobulin. The Wassermann reaction was negative. No virus could be isolated from plasma or CSF. A routine viral screen was entirely normal and a rubella antibody test showed a haemagglutination inhibition titre of $1 / 256$, with a complement fixation titre of less than 8 and a negative $\operatorname{IgM}$ sucrose density grading fractionation test. The patient was treated with prednisolone $80 \mathrm{mg}$ daily for three days, which was then reduced to $40 \mathrm{mg}$ for the following week. Over the next six weeks she made an excellent recovery.

She was seen again in the clinic six months later, this time complaining of pain on moving her left eye for the previous two weeks. She was found to have a left optic neuritis, decreased visual acuity of $6 / 60$ in the left eye compared with $6 / 12$ in the right, and a left large centrocaecal scotoma. The ESR on this occasion was normal and a lumbar puncture showed clear CSF without cells, protein content $0.52 \mathrm{~g} / 1$, and IgG $10.5 \%$. Attempts at vira isolation were negative and the rubella antibody titre was $1 / 256$. Routine tests for viral antibodies were normal. She was treated with intramuscular tetracosactrin $1 \mathrm{mg}$ daily for one week. She was seen again four months later when she had made an excellent recovery, the only abnormality being a little pallor of both optic discs.

Eight months after the second attack she had a further attack of paraesthesiae involving the two legs and on examination was found to have mild paraparesis, increased reflexes in both legs with bilateral extensor plantar responses, and pain and temperature sensory loss to T7 on the left and T5 on the right side. On this occasion she stated that her symptoms began one week after exposure to children with rubella. A lumbar puncture was not carried out. She was treated with a similar dose of tetracosactrin and made an excellent response once again over the ensuing six weeks. Viral studie on this occasion showed a rubella antibody titre of $1 / 128$, a complement fixation titre of less than 8 and no detectable antirubella $\mathrm{IgM}$. A routine vira antibody screen was negative. The patient has remained symptom-free since then.

This patient has a syndrome which seems indistinguishable from multiple sclerosis An attack has been precipitated twice by exposure to rubella virus, on the first occasion by vaccination and on the second by coming into contact with children who had rubella. Her illness responded to steroids. There were no abnormal findings apart from a raised ESR with a slight CSF pleocytosis in the first attack and a mildly raised CSF protein in the second. Failure to isolate the virus in no way militates against the disease being caused by a virus, though in virtually all cases of postinfectious encephalomyelitis no virus has been isolated. This finding has strengthened the hypothesis that post- or para-infectious encephalomyelitis represents a remote complication of infection -that is, immune-mediated demyelination. In support of this has been the demonstration of cell-mediated immunity to myelin encephalitogenic basic proteins in patients with acute disseminated encephalomyelitis. ${ }^{1}{ }^{2}$ How ever, in opposition to this view has been the isolation of measles virus ${ }^{3}$ and the demonstra tion of inclusion bodies in patients with post-measles encephalomyelitis. ${ }^{4}$ Postinfectious encephalomyelitis with post-mortem verification has previously been described following rubella, ${ }^{56}$ and indeed multiple sclerosis itself may occur following vaccination against several viral diseases. ${ }^{7}$ The immunopathological mechanisms by which involve ment of the nervous system occurs following vaccination is far from clear. It may well represent latent virus in cells which are attached by sensitised lymphocytes or humoral antibody or alternatively sensitisation to neural constituents.

Whether or not this patient suffers from relapsing acute disseminated encephalomyelitis or multiple sclerosis is a moot point and one that cannot be solved to date. There are points clinically in this case that favour both diagnoses and, indeed, pathologically verified cases have been described in which both diseases occured. ${ }^{8} 9$ The differences between the two diseases more likely result from genetic and immunological factors which govern the patient's reaction to the same agent. "Overlap" syndromes are therefore likely to occur. The further documentation of this association of diffuse demyelinating disorder with vaccination may help in our understanding of the cause of multiple sclerosis.

Peter O Behan

Institute of Neurological Sciences,

Southern General Hospital,

Glasgow

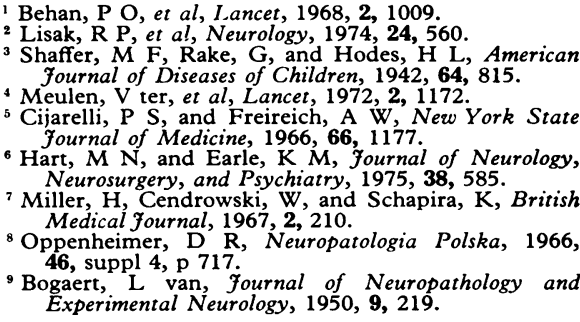

\section{Pancreatic diagnosis}

SIR,-In their recent comparison of pancreatic isotope scans, pancreatography, and Lundh tests Dr C J Mitchell and his colleagues (27 November, p 1307) found that a normal scan was strong evidence against pancreatic disease and recommended the scan as a screening test. In this unit with a particular interest in pancreatic diagnosis results and conclusions have been different. Our previous experience had been disappointing, but we set out here to make an assessment in collaboration with the department of nuclear medicine. Among 81 patients undergoing careful isotope scanning there were 30 with unequivocably normal pancreatic images. Twelve $(40 \%)$ proved to have definite pancreatic disease (two cancer, five severe chronic pancreatitis, five relapsing pancreatitis). Among 40 patients finally judged to have no pancreatic disease, only 16 had an unequivocally normal scan; 11 scans were abnormal and 13 equivocal. There were a total of 28 patients with unequivocally abnormal scans. Eleven (40\%) were finally judged to be free of pancreatic disease. Thus in this series scans had $40 \%$ false-positive and $40 \%$ false-negative results. We concluded that scans were of no clinical value and no longer use them.

There have been other recent developments. While computed tomography could not be regarded as a screening test, we have been impressed by the recent results of grey-scale ultrasonography, a technique which is quicker, cheaper, and presumably safer than isotope scanning.

However, at present when attempting the diagnosis of a patient who may have pancreatic disease we rely most heavily on clinical judgment and endoscopic pancreatography (ERCP). While the latter technique is complex and has potential hazards, these can be overcome. We have provided an adequate pancreatogram in $92 \%$ of our last 200 cases, with 
no complications. An abnormal pancreatogram absolutely defines pancreatic disease. There are some difficulties in interpretation of very minor changes and in distinguishing obstruction due to cancer or pancreatitis, at least when clinical information is withheld. A normal pancreatogram does not completely exclude cancer. We have seen one patient with a normal main duct radiograph (with poor branch filling) among 55 cases of pancreatic cancer. Equally, a normal pancreatogram does not exclude an early stage of chronic pancreatitis, as judged histologically. Here, however, the clinical picture is usually one of recurrent pain with a raised serum amylase. The diagnosis is already made and a normal pancreatogram, far from being misleading, is helpful in protecting the patient from pancreatic surgery. Conversely, an abnormal pancreatogram provides a map on which to plan surgical triumphs.

When faced with a patient with obscure upper abdominal pain we are concerned for the diagnosis and exclusion of peptic ulcer disease, gall stones, gastric and pancreatic cancer, and chronic pancreatitis. In this context ERCP (which includes gastroduodenoscopy, retrograde cholangiography, and pancreatography) is a powerful tool.

\section{P B CotTon R B STERN} Gastrointestinal Unit, Middlesex Hospital
London W1

\section{Lomotil in acute diarrhoea}

SIR,-In his article on this subject (20 November, p 1240) Dr D R Bell has given a paragraph to diphenoxylate with atropine (Lomotil) which may be misleading.

As he so rightly says, Lomotil is popular nowadays. This is because it is found to be effective and commonly free from side effects. Like most other drugs of therapeutic value, it has unwanted additional actions, but these are not frequently encountered. Warnings are provided that Lomotil should not be given with sedatives and cerebral depressants, but one wonders where he derived his information that "it can potentiate the effects of alcohol dramatically."

The broad statement that the drug "is no longer recommended for children" is unacceptable. It must be asked, On whose recommendation? How old are the children? What are the facts in support? There are no references at the end of the article. The Committee on Safety of Medicines accept its use in children from 1 year of age.

If the drug has the dangers he implies Dr Bell should have been as precise about dosage as the manufacturers. Equally as important, the drug is not related to morphine but to pethidine and there is no evidence that long-term administration leads to habituation. When overdosage does occur with respiratory depression the drug of choice in treatment is naloxone.

Maurice CoHeN Medical Director
Searle Laboratories High Wycombe, Bucks

${ }^{*} *$ We sent a copy of this letter to Dr Bell, whose reply is printed below.-ED, BMF.

SIR,-Of course it is true that Lomotil is commonly free from side effects, but a responsible review of current practice cannot afford to ignore side effects that do sometimes occur. The statement that Lomotil "can potentiate the effects of alcohol dramatically" is based on personal observation and is intended to warn against giving Lomotil with alcohol or any other central nervous system depressant drug. The manufacturer's literature recommends "caution" under these circumstances; I think my recommendation to avoid such combinations is more helpful.

The use of Lomotil in children below the age of 12 is a matter for the physician's own discretion. My article was intended to reflect the consensus of current medical practice in Britain and I believe I have done this.

That prolonged use of diphenoxylate can cause dependence of the morphine type is stated in Martindale's Extra Pharmacopoeia. ${ }^{1}$ The same source also refers to a report of coma and cardiac arrest in a 2-year-old child after ingestion of as few as six Lomotil tablets. ${ }^{2}$

I agree that the manufacturer's 1973 recommendation of nalorphine as the preferred antidote to diphenoxylate poisoning has now been superseded and that naloxone, being free of depressive effects itself, is the drug of choice.

\section{Department of Tropical Medicine Liverpool School of Tropical Medicine,} Liverpool

'Martindale: The Extra Pharmacopoeia, ed N W Blacow, 26t

${ }^{2}$ Henderson, W, and Psaila, A, Lancet, 1969, 1, 373.

\section{The hospitals we need}

SIR,-Dr R M Emrys-Roberts has summarised the Oxford cost-effectiveness study concisely (4 December, $p$ 1385). The thoroughness of the Oxford experiment deserves wide recognition and it is sad that our colleagues there have had to give the financial kiss of death to their brainchild so early in the life of the community hospital. What is not clear is why the Department of Health and Social Security accepted this experiment as the blueprint for their own discussion document. ${ }^{1}$ Experience of some 350 general practitioner hospitals containing some 9000 beds, much of it now fully recorded by Hospital Activity Analysis, has been available for some time. The scope and costs of providing hospital services in small units could usefully have been subjected to open debate before any discussion document was produced.

The main difference between the more active GP hospitals and the Oxford Community Hospital Programme is one of emphasis on the hospitals' acute role. Arbitrary limitation of this aspect of hospital work is likely to lead to a severely limited service to the community in question and subsequent loss of cost-effectiveness. In Brecon the casualty:admissions ratio is $5: 1$, and the $x$-ray:admissions ratio is $3: 1$. The casualty department is the busiest and relatively most efficient in the hospital, with a referral rate to district general hospitals of less than $10 \%$. This compares with an inpatient referral rate of $30 \%$ to $\mathrm{DGH}$ from the community as a whole, $70 \%$ being treated in Brecon Hospital. Transfer costs for patients in the Oxford area were a significant cause of loss of cost-effectiveness. The more acute services that are provided locally, the less this factor will predominate.

The chief loss in the debate so far has been the potentially useful term "community hospital," which has been elevated to a great height only to be shot down on financial grounds-the most devastating of all at the moment. The Association of General Practitioner Hospitals is about to produce a document in similar terms to those of the DHSS for further discussion. Could not the name "community hospital" be viewed afresh from the time of its publication?

\section{A J M Cavenagh}

Brecon, Powys

Department of Health and Social Security, Community
Hospital. Their Role and Development in the National
Health Service. London, HMSO, 1974.

\section{SI: two years on-a lesson for the \\ Royal Commission}

SIR,-I share the lack of enthusiasm in your leading article ( 1 January, $p$ 5). Molar concentrations are largely irrelevant to clinical medicine-I can count on my toes the times I have used them in clinical decisions in the past two years. If I want the plasma osmolality I do not add six experimental errors and a few false assumptions: I measure it.

New graduates acquire facility with the new system as we did with the old, but for my generation the switch to SI wiped out, at a stroke, 25 years of experience in interpreting results. Two years later I respond emotionally to creatinine and urea in SI units but I still convert dextrose, calcium, phosphate, urate, bilirubin, and blood gases into "old units" before making decisions. This was entirely predictable by anyone who had watched the more necessary introduction of multiequivalent $\mathrm{mEq}$ for the common electrolytes 20 years earlier. We were told that the transition to SI would be quick and we would soon be wondering what all the fuss was about, but for those of us who have 10-20 years' clinical practice ahead and know we will never be as sharp with the new units as we were with the old the transition is pestilentially slow.

At the outset I wrote to Sir George Godber protesting against the new system and urging him at least to preserve the $\mathrm{mm} \mathrm{Hg}$ for blood pressure. I received a bland reply from one of his colleagues saying there were no major problems with the changeover; I could only conclude that he never treated patients or read the $B M F$ and the Lancet. Had he been brainwashed by a handful of biochemists and failed to realise that laboratories can express their results in ells and furlongs at the touch of an electronic calculator, but they are interpreted by clinicians? We were assured that $20+$ official bodies had been consulted, but the Department of Health and Social Security, having created Cogwheel, never used its own system to sound the opinion of those who would have to use SI in their daily work.

It is this lack of contact between the Elephant and Castle and the front line of medicine that I hope the Royal Commission will notice. Some decisions (including the future of SI) have to be made centrally but a lot more could be devolved, provided resources are shared fairly between regions and cash limits are applied impartially across the country. If these objectives are achieved is it really necessary for the minutiae of building plans, painstakingly drawn up with local consultation, to be rescrutinised in London? Indeed, does the DHSS need to be involved at all in decisions about the siting and size of hospitals or health centres? Need we have uniform policies across 